\title{
LA INFORMACIÓN PERIODÍSTICA Y LA INVESTIGACIÓN DEL RIESGO AMBIENTAL
}

\author{
Claúdia E.Natenzon *
}

\begin{abstract}
The application of the social theory of risk allows the examination of environmental complex problems identifying several perspectives from which they can be analyzed. These perspectives include hazard, exposition, vulnerability and uncertainty. One of the main objectives needed to inquiry on the aspects related to vulnerability and uncertainty, is to detect for whom and in which conditions a natural phenomena can be considered as «catastrophic» To meet this goal, the production of news from mass media can serve to the objectives of a research activity, as an exploratory informant and a first approach to the problem. In this sense, some precautions have to be taken in order to improve the information from the level in which it is produced - dissemination and common sense- To be rigorous, we consider insufficient the citation of the source; additional aspects should be taken into account: Identify precisely the universe of news reports to analyze; assess the importance of the news report in the selected media; identify the published information; and characterize the selected media as an actor that is not neutral. Although the analysis of news reports is aimed to both, quantitative and qualitative aspects, the final result is eminently qualitative.
\end{abstract}

\section{PALABRAS CLAVE:}

Investigación. Riesgo ambiental. Comunicación social. Periodismo.

\section{Introducción}

Con la finalidad de considerar integralmente un fenómeno físico-natural con consecuencias catastróficas, la adopción de una teoría social del riesgo nos ha permitido identificar varias perspectivas necesarias para su análisis (Natenzon, 1995): respecto a la causalidad de los procesos físico-naturales - peligrosidad- respecto a las configuraciones territoriales -exposición- respecto a la estructura socio-económica antecedente vulnerabilidad- y respecto a las acciones, prácticas, políticas y percepciones de la sociedad involucrada -incertidumbre-

Para indagar sobre los aspectos que hacen a la vulnerabilidad y la incertidumbre, uno de los objetivos centrales de nuestras investigaciones es reconocer para quién y en qué condiciones los fenómenos «naturales» pueden ser calificados de catastróficos, tomando en cuenta las condiciones heterogéneas previas de los grupos sociales involucrados (Natenzon, 1993). Una parte de este reconocimiento incluye establecer qué concepciones están instaladas en el sentido común de la sociedad respecto a un fenómeno ambiental particular, en nuestro caso, las catástrofes «naturales».

La prensa escrita se constituye en una fuente de información privilegiada para este fin. La generación de noticia por parte de un medio masivo de comunicación social

* Pirna Programa de Investigaciones en Recursos Naturales y Abiente, Facultad de filosofía y Letras, UBA/ FLACSO 
puede funcionar, a los fines de la tarea de investigación, como informante exploratorio que brinda una primera aproximación al problema de análisis, siempre y cuando este uso cumpla con algunas condiciones mínimas.

\section{Condiciones para el uso de material periodístico}

El análisis del material periodistico puede ser abordado de distintas maneras.

Se puede tomar a la práctica periodística como objeto de análisis en si, dando pautas sobre el «estado del arte» en esta forma literaria (Fernández Sánchez, 1995); el análisis del discurso se realiza aplicando al texto periodístico marcos conceptuales y técnicas provenientes de la crítica literaria, la lingüística y la semiótica (Atorresi, 1995).

- Desde el punto de vista de la sociología y la ciencia política, se analiza la producción periodística en tanto expresión de un agente/ actor/ sujeto -el de la prensa escrita como medio masivo de comunicación-: sus relaciones con el poder y las mediaciones que produce entre la información y la sociedad civil (Sidicaro, 1997).

El conocimiento etnográfico requiere construir datos primarios, para lo cual los diarios son una fuente, en situaciones donde resulta imposible mantener contacto «en el campo». por ej., con personajes públicos centrales, determinantes $y$ portadores de la lógica que impregna la toma de decisiones politicas (Grassi, 1994).

La presente propuesta de trabajo se inscribe principalmente en el último de estos abordajes, tomando de los otros algunos aspectos complementarios. Aquí se apunta a desentrañar cómo se caracteriza al evento tomado como «noticia», las consecuencias socio-económicas puestas de manifiesto y consideradas relevantes por el medio de prensa elegido, que es tomado como expresión de aspectos, circunstancias, perspectivas, etc. de interés para la opinión pública, para la sociedad en su conjunto y para el propio medio de comunicación en tanto -también él-actor social.

En este sentido, resulta necesario tomar recaudos para que la información no quede en el nivel en el que es producida: el de la divulgación y el sentido común, dándole cierta rigurosidad a través de pautas metodológicas que transparenten y sistematicen la forma en que llegamos a determinados resultados. Para ello consideramos que no es suficiente citar la fuente utilizada (recaudo mínimo de rigor) sino que es necesario tomar en cuenta y desarrollar aspectos adicionales:

Identificar con precisión el universo de noticias a analizar.

Evaluar la importancia dada a la noticia en el medio elegido.

Identificar la información publicada, cualitativa (los temas, los actores) y cuantitativamente (las cifras publicadas).

Caracterizar al medio de prensa elegido, en tanto actor del proceso de comunicación del riesgo.

\subsection{Universo de noticias a analizar}

El universo a estudiar se refiere a la expresión impresa del fenómeno ambiental (para nosotros, aquellos de tipo catastrófico) considerado como noticia. La «noticia» es el hecho a transmitir. Hay noticia siempre que ocurra algo -un evento novedoso- que se pueda contar.

Dicho universo ha de ser revisado minuciosamente y fichado, tomando como unidad a cada uno de los «artículos» o «notas» que el medio de prensa elegido editó sobre el evento o fenómeno en cuestión, a partir de la primer fecha en que apareció una referencia al tema en el cuerpo principal del diario, de manera continua o discontinua hasta que ya no aparece nota alguna por un lapso continuado mínimo, lapso que deberá definirse según los objetivos planteados en cada investigación (por una semana, un mes, un semestre, un año).

Se define como «artículo» o «nota» a un texto diferenciado del resto por su coherencia interna propia, que se edita en un periódico, un día y una sección determinados. La noticia puede estar constituida por varios artículos agrupados en una misma sección o dispersos en distintas secciones del diario. Una forma especial de artículo o nota es aquella llamada «de opinión», en donde se identifica al autor por su firma. Otra forma especial de artículo - nota es cuando se reproduce un cable textualmente, tal como se recibió de una agencia 
de noticias externa a la redacción del diario.

El fichado apunta a construir una base de datos, que puede estar formada por campos de información tales como los siguientes: nro. de orden del registro, nombre de la publicación fecha de publicación (día, mes, año), número de página/s en donde se encuentra el artículo, sección del diario, extensión (superficie del artículo en $\mathrm{cm} 2$.), autor, agencia de noticias -si corresponde-, título, ante título/s -si corresponde-, inclusión de gráficos (fotos, esquemas, cuadros, cartogramas, mapas, tablas), y sintesis de los principales temas considerados, donde se puede reproducir la «bajada» o «copete» (que sintetiza las principales líneas temáticas de artículos muy extensos), referencias textuales $y / 0$ comentarios propios sobre los principales aspectos contenidos en el artículo.

El fichado permite una primer lectura de la noticia a lo largo del tiempo. Una vez concluida esta primera sistematización se deberán realizar múltiples y sucesivas lecturas transversales al texto, con sus correspondientes registros; tantas lecturas como aspectos particulares se desee identificar. El resultado es reordenado en aspectos cuantitativos (importancia dada a la noticia en el período considerado; tipo de información cuantitativa utilizada en las noticias) y cualitativos (temas centrales que recorren las noticias del fenómeno y del periodo considerado, agentes/ actores/ sujetos sociales que el medio periodístico identifica vinculados a la noticia).

\subsection{La importancia de la noticia}

La importancia dada a la noticia en el período considerado es analizada partiendo de suponer que hay una relación directa entre la superficie asignada a los artículos y el interés para el periódico/el público, pues cada centímetro cuadrado tiene para el medio un valor. También es importante examinar la continuidad/ discontinuidad de la publicación de esa noticia en el lapso considerado.

Para evaluar esta importancia se puede medir la superficie de cada artículo, incluyendo su aparición en tapa, acumulando el valor para cada día de publicación. El resultado se indica como valores absolutos de superficie, en $\mathrm{cm}^{2} /$ día. También se puede establecer un segundo valor, de tipo relativo, en tanto por mil de la superficie total de cada ejemplar del diario (superficie relativa $=$ $1000 \times$ superficie absoluta / (nro. de páginas $x$ superficie de una página, en $\mathrm{cm}^{2}$ ). La secuencia puede ser representada gráficamente, mostrando las variaciones de superficie $y$, en consecuencia, del interés evidenciado por la noticia. Sus fluctuaciones deberán ser analizadas e interpretadas a la luz del desarrollo del propio fenómeno, de sus consecuencias inmediatas y a largo plazo, y del contexto socioeconómico en el cual dicho fenómeno tiene lugar.

Por ejemplo, en la aplicación de esta forma de trabajo para el caso de la erupción del $\mathrm{Vn}$. Hudson (Natenzon, 1996) el gráfico resultante de esta tarea permitió observar un desfasaje de seis días entre el momento de la primer erupción ( 8 de agosto de 1991) y la aparición de la primer noticia sobre el tema, lo que hemos atribuido a que la espectacularidad del evento y sus consecuencias catastróficas sólo se hicieron presentes con la segunda erupción (12 de agosto de 1991). Luego, la noticia se mantuvo vigente durante 17 días continuos, de manera decreciente durante la primer semana, hasta estabilizarse a valores muy bajos en la segunda, pero a mediados de septiembre hubo una reaparición, una reinstalación de esta noticia, lo que no debe atribuirse a nuevas erupciones ni a nuevas consecuencias catastróficas (hasta esa fecha las lecturas temáticas habían agotado la casi totalidad de tópicos o aspectos en discusión), sino a la aparición de otra noticia "ecológica" de tipo catastrófico en la misma región -pingüinos empetrolados en la costa atlántica-, con la cual se la vinculó.

\subsection{Información cualitativa; los temas, los actores}

Este punto expresa los temas más relevantes que constituyen la noticia. Han perdido el orden cronológico (aparición en la secuencia de la lectura) y han sido reordenados en función de un nuevo criterio, el de los temas tratados y su desarrollo particular. Las sucesivas lecturas han permitido su identificación, produciéndose en algunos casos un «efecto de saturación», por el cual las mismas cuestiones son repetidas una y 
otra vez a medida que se avanza en el tiempo, y llega un punto en el cual ya no se encuentran cuestiones nuevas más que esporádicamente.

Al mismo tiempo, el análisis requiere identificar a los actores valorados para la noticia: A quiénes se interroga, quiénes dan opinión, qué pobladores son entrevistados, qué autoridades políticas y qué expertos visitaron el área; en qué circunstancias lo hicieron, con qué finalidad y qué dijeron. No necesariamente todos los que aparecen son importantes y no todos los importantes necesariamente aparecen.

Por ejemplo, en un trabajo que realizamos sobre las inundaciones de carácter catastrófico en la cuenca del río Salado en la Provincia de Buenos Aires, ocurridas en 1993 (Natenzon et al, 1997) señalamos, entre otros aspectos, quiénes participan del debate sobre la cuestión agraria y qué postura asumen ante las inundaciones. Para tal fin se efectuó un análisis de notas periodisticas publicadas en los diarios Clarín, La Nación y Página 12 entre los meses de marzo y junio de ese año. Se realizó una identificación de la forma en que los medios de comunicación abordaban esta problemática, especialmente en lo referido a cada agente social involucrado y a las vinculaciones establecidas entre ellos: los productores agrupados (CARBAP, Federación Agraria Argentina, Sociedad Rural Argentina), distintas instancias del gobierno en el ámbito nacional (SAGyP, INTA, Ministerio de Economía, Ministerio del Interior), instancias del gobierno en el ámbito provincial (Dirección de Hidráulica, Ministerio de la Producción, Ejecutivo provincial, Banco de la Provincia), gobiernos en el ámbito local (distintas intendencias afectadas). En cada caso primero de describió la postura registrada por la prensa y luego se realizó una interpretación de dichas posturas a la luz de nuestro marco teórico.

Estas identificaciones también permiten realizar listados de informantes a ser entrevistados en el trabajo de campo. A la vez pueden reconocerse las propuestas de acción realizadas, en qué medida se van concretando en el tiempo y con qué grado de efectividad, siempre según el medio de prensa analizado.

\subsection{Información cuantitativa}

Otro aspecto a considerar es la importancia dada a la información cuantitativa en la configuración de la noticia. Esto puede realizarse identificando qué datos se incluyeron, con qué frecuencia y qué consistencia mantienen entre sí. Para analizar este punto se puede construir un cuadro de doble entrada utilizando alguna de las planillas de cálculo estándar, en donde la primer columna registre sistemáticamente los temas considerados, desarticulados en ítems lo más específicos posible; y las columnas siguientes, las cifras publicadas día por día en orden cronológico.

Por ejemplo, en el caso citado del Vn. Hudson se identificaron 76 temas, desplegados en más de 200 ítems, mostrando una abundancia de cifras excepcional: coordenadas geográficas del volcán, número de habitantes de los asentamientos afectados, distancia entre ellos, fechas de erupciones anteriores, visibilidad en distintos sitios, espesor de ceniza acumulada y superficie cubierta, altura de la columna de humo, número de cráteres, diámetro de cada uno, número de cabezas de ganado afectado, número de evacuados (por asentamiento, por sexo, por edad), ayudas y donaciones, subsidios, créditos, pérdida económica, tiempo que demorará la recuperación, tiempo escolar perdido, costos (de fletes, de vida en cada asentamiento), rentabilidad, etc. etc.

El aporte de datos en las noticias de este tipo es permanente, y su análisis evidencia cierto grado de inconsistencia y falta de rigor en el uso de las cifras; por ej., para el mismo ítem, se publican datos distintos, o se incluyen datos sin citar la fuente consultada.

Se obtendrá, de esta manera, un panorama de tipo cualitativo con relación a cuáles son los datos y las fuentes priorizadas por la prensa.

\subsection{Caracterización del medio de prensa}

Los medios masivos de comunicación juegan un rol central en el proceso de comunicación del riesgo. Ellos dejan registro escrito de lo que distintos sectores de la sociedad piensan $y$ hacen sobre el riesgo en cuestión, transmiten al público información múltiple $y$, a la 
vez, ejercen una notoria influencia en la configuración de lo que la opinión pública considera o no como riesgo ambiental.

Una empresa mediática no es un actor neutro:

«...no tanto el producto pero si las entidades productoras son hijas de un cierto estado del capitalismo, de sus frutos tecnológicos y, más importante aún, de una cierta manera de interpretar al mundo y las relaciones entre los hombres» (Traversa, s.d.f., 17).

Un medio de prensa no es un actor coherente y monolítico. Sus páginas reflejan opiniones múltiples de distintas voces (Sidicaro, 1997).

Algunos datos básicos para tomar en cuenta son el tiempo de permanencia que tiene el medio en el mercado, las características de los capitales que la conforman, el tipo de empresa en cuanto a medios de comunicación incluidos (monomediática, multimediática), el número de ejemplares editados, el público al que está dirigido y el público que lo adquiere, la escala de su distribución (local, provincial, regional, nacional), la frecuencia de aparición (diario, periódico), la estructura y organización interna de cada ejemplar, las secciones en las cuales pueden aparecer los temas de interés.

En Argentina existen varios diarios publicados en la Capital Federal, que se difunden en ciudades capitales del interior del país, no especializados -La Nación, Clarín, Página 12- y especializados -Ámbito Financiero y El Cronista Comercial- En el ámbito provincial se publican diarios con cierta tradición, que pueden tener impacto a nivel regional. Antes de la difusión de medios masivos de comunicación tales como la televisión $y$, en la última década, los medios electrónicos (Internet), existía un número importante de periódicos publicados en el ámbito local, que fueron reduciéndose y a los que prácticamente les resulta muy difícil tener continuidad, como consecuencia de la crisis económica de la última década.
Cada uno de ellos (como espejo del escenario en el cual se desarrolla el riesgo en análisis) presentará la noticia desde múltiples perspectivas, como reflejos aproximados de las múltiples perspectivas que portan los actores involucrados. Cada uno de ellos (como actor involucrado) apuntará a que determinados aspectos del riesgo y no otros formen parte de la opinión pública sobre el tema. Por esta razón el mismo esquema de análisis debería ser aplicado a múltiples medios de difusión periodística, en las escalas consideradas relevantes para cada problemática ambiental particular: local, provincial, nacional, internacional.

\section{El uso de la información obtenida}

Si bien el trabajo de análisis o disección planteado apunta tanto a los aspectos cualitativos como cuantitativos que aparecen en las noticias, el resultado final es de corte eminentemente cualitativo.

Debemos dejar constancia que aquí no se avanza en discutir cuán pertinentes, válidos, oportunos y veraces son los datos y las consideraciones realizadas en los artículos periodísticos sobre el fenómeno según los parámetros establecidos por la academia. Para complementar este ejercicio, resultará necesario identificar la calidad de la información difundida comparando los resultados obtenidos con los protocolos establecidos en las ciencias que estudian el problema considerado.

El producto obtenido, en algunos casos, puede llegar a constituirse en la única fuente de información disponible. Al mismo tiempo, un análisis de esta naturaleza también es útil para los procesos de comunicación social de las ciencias ambientales, a fin de detectar el reflejo del accionar académico y profesional en la sociedad civil, observar la forma en que se difunde el conocimiento científico (con aciertos, falacias o deformaciones) en el ámbito público $y$, finalmente, pensar en un rol más eficaz de los científicos para la transferencia, la divulgación y la capacitación. 


\section{Bibliografia}

Atorresi, Ana (1995) La crónica periodística. Buenos Aires, Ars Comunicación y Cultura; 160p.

Atorresi, Ana -editora- (1995) Los géneros periodísticos. Buenos Aires, Colihue; 200p.

Fernández Sánchez, Joaquín (1995) Periodismo Ambiental en España. Madrid, MOSPTyMA. Serie Monografías; $132 \mathrm{p}$.

Grassi, Estela (1994) «La antropología de lo político en la sociedad moderna: una propuesta de construcción de los datos de campo a partir de la información contenida en los medios de prensa» En: Primeras Jornadas sobre Etnografía y Métodos Cualitativos. Buenos Aires, 9 y 10 de junio de 1994 (mimeo).

Natenzon, Claudia E. (1993) «Processos catastróficos no nordeste argentino. Mudanças produzidas na última década». En: O Novo Mapa do Mundo. Naturaleza e Sociedade de Hoje: Uma Leitura Geográfica. São Paulo, HUCITEC/ ANPEUR (182-195)

Natenzon, Claudia E. (1995) Catástrofes naturales, riesgo e incertidumbre. Buenos Aires, FLACSO.
Serie Documentos e Informes de Investigación Nro. 197

Natenzon, Claudia E. (1996) «Volcanes y comunicación social». En: Riesgo volcánico. Islas Canarias, Centro Internacional de Volcanología y Geofísica Volcánica/ CSIC/ UNESCO. Serie Casa de los Volcanes Nro. 5 (223-244).

Natenzon, Claudia E. et al. (1997) Inundaciones, producción agropecuaria y agentes sociales de la cuenca del río Salado, Provincia de Buenos Aires. Gorizia, Programma Emergenze di Massa/ Istituto di Sociologia Inernazionale. Quaderno n.97 - 4; $19 \mathrm{p}$.

Sidicaro, Ricardo (1997) «Consideraciones a propósito de las ideas del diario La Nación». En: La Trastienda de la Investigación. C Wainerman y R. Sautu, comp. Buenos Aires, de Belgrano; Cap.3. (73-90).

Traversa, Oscar (s.d.f.) «El fin de las noticias» En: Télam. Medio siglo de noticias. Buenos Aires, [1995?], (17). 